\title{
Thermal and Structural Performance of Woven Carbon Cloth \\ For Adaptive Deployable Entry and Placement Technology
}

\author{
James O. Arnold ${ }^{1}$ \\ NASA Ames Research Center, Moffett Field, CA 94035 \\ Keith H. Peterson ${ }^{2}$ \\ ERC, Inc. at NASA Ames Research Center, Moffett Field, CA 94035 \\ Bryan C. Yount, ${ }^{3}$ \\ NASA Ames Research Center, Moffett Field, CA 94035 \\ Nigel Schneider ${ }^{4}$ \\ Northrup Grumman (TEAMS2) at NASA Langley Research Center, Hampton, VA 23681 \\ and \\ Jose Chavez-Garcia ${ }^{5}$ \\ ERC, Inc. at NASA Ames Research Center, Moffett Field, CA 94035
}

Arcjet testing and analysis of a three-dimensional (3D) woven carbon fabric has shown that it can be used as a thermal protection system and as a load bearing structural component for a low ballistic coefficient hypersonic decelerator called ADEPT (Adaptive Deployable Entry and Placement Technology). Results of arcjet tests proved that the 3D woven carbon fabric can withstand flight-like heating while under flight-like biaxial mechanical loads representative of those encountered during shallow entry flight path angles into the atmosphere of Venus. Importantly, the arcjet test results have been used to extend a preliminary material thermal response model based on previous testing of the same 3D woven carbon fabric under uni-axial mechanical loading.

Nomenclature

$B^{\prime}{ }_{C}=$ Outgoing mass flux of carbon divided by incoming free stream mass flux, dimensionless

$C_{H}=$ Convective heating film coefficient, $\mathrm{kg} / \mathrm{m}^{2} \mathrm{~s}$

$R A=$ Recession augmentation factor, dimensionless

$S_{\mathrm{dot}}=$ Recession rate, $\mathrm{m} / \mathrm{s}$

$q_{c}=$ Convective heat flux, $\mathrm{W} / \mathrm{m}^{2}$

$\rho \quad=\quad$ Carbon cloth density, $\mathrm{kg} / \mathrm{m}^{3}$

\section{Introduction}

Work on the Adaptive Deployable Entry Placement Technology (ADEPT) began in October 2011 and is funded by the NASA Headquarters Space Technology Mission Directorate (STMD), Game Changing Development Program. Figure 1 shows an ADEPT entry system resembling an umbrella that is stowed for launch within the rocket's shroud and deployed in space prior to atmospheric entry. Envisioned for use in a future mission that would deliver the Venus Intrepid Tessera Lander (VITaL)[1], the drawing depicts the ADEPT "skeleton" in both the stowed and deployed states. A major advantage of using ADEPT for the VITaL mission is that its reduced ballistic coefficient results in deceleration forces ten times lower than a conventional rigid body

\footnotetext{
${ }^{1}$ Senior Scientist, Entry Systems and Technology Division, Mail Stop 229-3, Fellow AIAA

${ }^{2}$ Computational Materials Scientist, Thermal Protection Materials and Systems Branch, Mail Stop 234-1, Member AIAA

${ }^{3}$ Aerospace Technologist, NASA Experimental Facility Development, Engineering Systems Division, Mail Stop 213-4

${ }^{4}$ Design Engineer, Mechanical Systems Branch

${ }^{5}$ Research Scientist/Engineer, Thermal Protection Materials and Systems Branch, Mail Stop 234-1
}

1

American Institute of Aeronautics and Astronautics 
capsule, thereby enabling the use of less robust, more capable scientific instruments for solar system exploration. In addition, the lower ballistic coefficient also enables deceleration to occur at higher altitudes with heating rates about ten times lower than those encountered by conventional rigid systems. This feature allows for consideration of a more efficient thermal protection system (TPS) for ADEPT, and was the reason for considering a 3-D woven carbon fabric for this function. Figure 1 also illustrates the 3-D woven carbon fabric that serves both as the TPS and the "skin" that transfers the aerodynamic deceleration forces to the ADEPT "skeleton". When deployed, the fabric is taut. The fabric is oriented with its warp direction connecting point-topoint locations at the same downstream coordinates on the ribs, while its weft direction runs outboard from the nose toward the trailing edge. For flight, bi-axial running loads are up to $131 \mathrm{kN} / \mathrm{m}$ in the warp direction and 66 $\mathrm{kN} / \mathrm{m}$ in the weft direction. Remarkably, analysis of the arcjet test data obtained in the NASA Johnson Space Center Test Point 2 (JSC TP2) facility [2] has shown that the carbon cloth thickness required for the 6-meter base diameter ADEPT entry system needed for the VITal mission is only $0.38 \mathrm{~cm}$. When deployed, the ADEPT entry system becomes a fairly flat decelerator with a 70 degree half-angle measured from the axis of symmetry capped by a blunt-body, rigid nose covered with a conventional TPS such as phenolic impregnated carbon ablator (PICA). ADEPT-VITaL will undergo a ballistic entry at an angle of attack of zero degrees.

Carbon fabrics can be woven in many different ways and the weave architecture is a significant factor in how it reacts to aerothermodynamic flows and tensile loading. Tensile testing on four candidate weaves for ADEPT characterized their relative mechanical performance [2]. This testing revealed that for each weave, hysteresis was present but could be significantly reduced by cycling it through several flight-like tensile loads. The focus of the prior arcjet testing at JSC was to select the carbon fabric weave from four candidates that gave the best relative performance during entry heating conditions for use as ADEPT's skin. This testing was conducted with the carbon cloth held in uni-axial tension, not representative of flight-like, bi-axial loading. Based on the relative aerothermal performance during the JSC arcjet testing and the said mechanical testing, a twelve-layer fabric was selected [2] for the ADEPT baseline fabric. This carbon cloth with twelve interlocked layers is called Super Weave A, or Weave SA for short. The twelve-layer carbon cloth is provided by Bally Ribbon Mills of Bally, PA. The Weave SA twelve-layer fabric is comprised of eight upper, sacrificial layers to provide the thermal protection system (TPS) while the lower four layers provide the structural function.

This paper presents test data and analysis of the ADEPT Weave SA under combined aerothermodynamic and flight-like bi-axial loading. The arcjet testing was conducted in the NASA Ames Interactive Heating Facility (IHF) and was entitled the Bi-axial Loaded Aerothermodynamic Mechanical (BLAM) test campaign. Air was used as the test gas in both the Ames and JSC [2] campaigns.

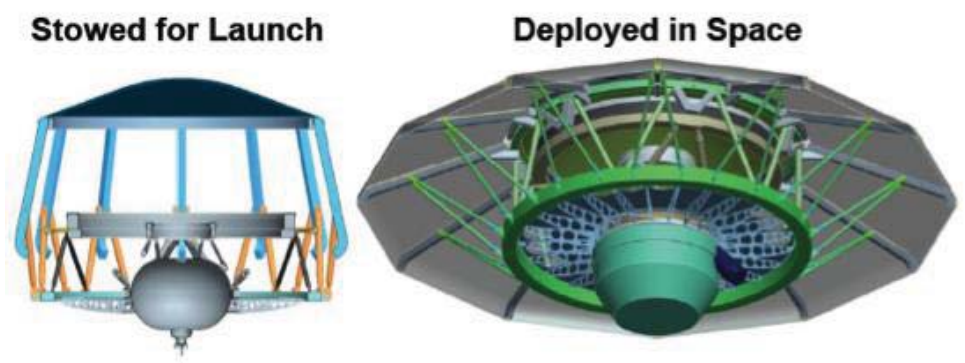

Figure 1. Depiction of a 6 meter diameter entry system that employs ADEPT for the Venus Intrepid Tessera Lander (VITaL) Mission [1]. (Left) stowed for launch and (Right) deployed in space prior to entry interface.

It was shown in reference [2] that the Fully Implicit Ablation and Thermal (FIAT) thermal response code [3] could be used to model the layer removal during aerothermodynamic heating of the carbon fabric, and this capability is extended to lower heating rates based on the BLAM data reported herein.

\section{Objectives of the BLAM Arcjet Test Campaign}

The primary test objectives of the BLAM arcjet test campaign were:

1. To evaluate the Super Weave A capability to maintain structural integrity under combined flight-like aerothermodynamic and flight-like bi-axial tensile loading.

2. To evaluate the rate of the fabric layer loss (recession) as a function of different aerothermal and biaxial loadings. 
Secondary test objectives were to provide arcjet tested fabrics to:

1. Evaluate the residual load bearing capacity of the arcjet-tested material.

2. Examine the microstructure of the arcjet-tested material using Scanning Electron Microscopy (SEM).

\section{Aerothermodyanamic Environments for ADEPT-VITaL}

\section{A. Predicted Flight Environments}

The goal of arcjet testing is to simultaneously replicate expected/predicted aerothermal environments pressure, shear stress, heat flux - encountered at a point on the flight article and directed at a point on a much smaller scale test article. Often, this goal is not possible to achieve owing to facility limitations. The time duration of an arcjet test is such that the test article experiences the same heat load (time-integrated integrated heat flux) as that of flight.

The upper part of Figure 2 depicts in frontal view contours of predicted pressures, hot-wall convective heat rate, and shear at the point of peak total heating (convective and radiative) along the reference trajectory for ADEPT during the VITaL mission. Predictions for laminar and turbulent flow are shown to the left and right on each frontal view, respectively. The computational capabilities of modern flow field simulation tools can be used to predict the aero/aerothermal environments on the depressed geometry (due to application of aerodynamic loads), and this was done for the results in Figure 2. . The lower part of Figure 2 depicts "butterfly plots" of heat rate versus pressure and heat rate versus aerodynamic shear at three body points - 10,12 and 14 -along the entry trajectory. The locations of these body points are shown on the head-on view of heat flux. The entry trajectory follows the butterfly curves in a clockwise direction, i.e., the time coordinate is along these curves with

time $=0$ at the origin. The predictions of heat flux are for the convective component, which is the dominant aerothermodynamic heating source. Aerodynamic shear at body point 14 increases as the flow approaches the corner radius as can be seen in the lower right portion of Figure 2. Total heating, including shock layer radiation, is predicted to be about 32 percent higher than the convective component at these points. 

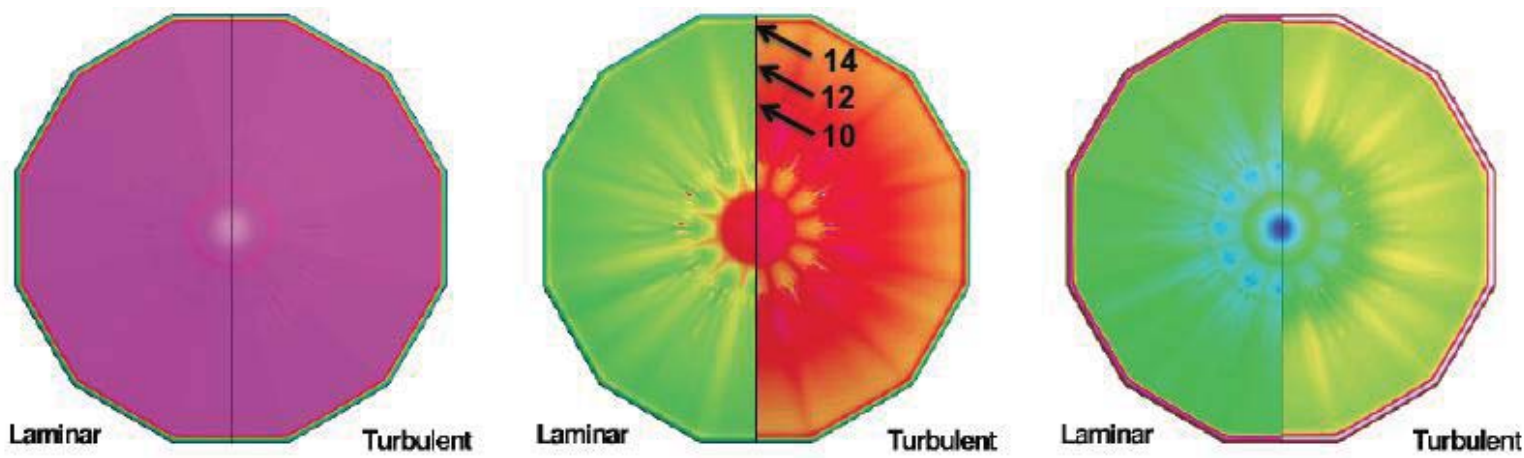

p, bar

$0 \quad 0.020 .040 .060 .08 \quad 0.1$

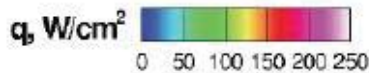

$\tau, \mathrm{Pa}$

$0 \quad 30 \quad 60 \quad 90120150$
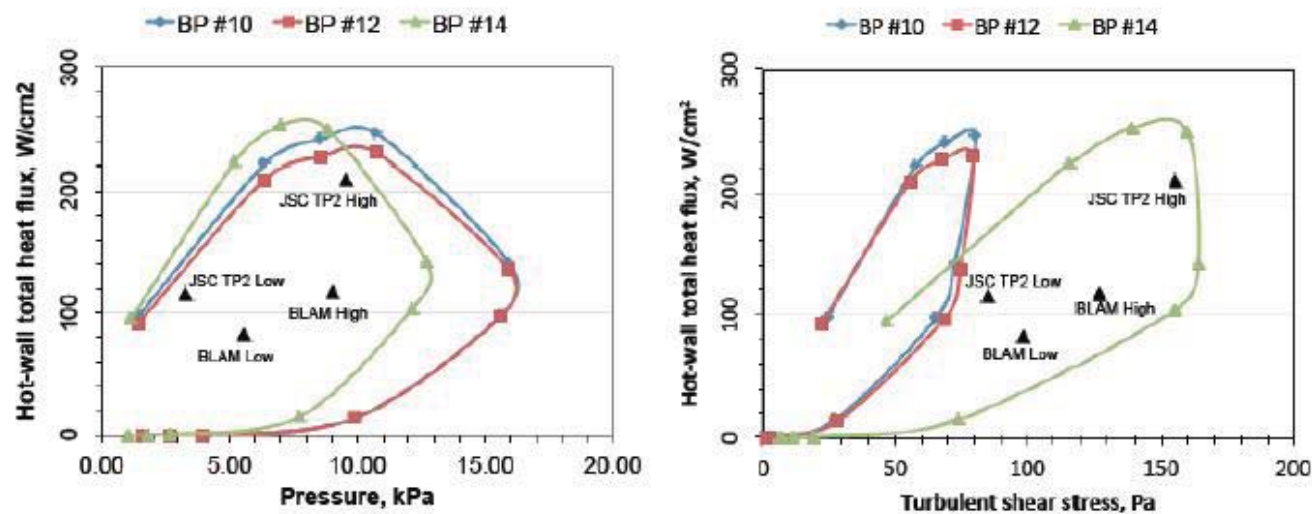

Figure 2. Hot-wall aerothermodynamic environments for the ADEPT entry system design for the VITaL mission. Fig. 2 (upper) depicts head-on views of predicted pressure, heat flux and aerodynamic shear accounting for "wrinkling" or depressions in the surface due to aerodynamic pressure loading at the peak convective heating point along the flight trajectory. Fig. 2 (lower) shows "butterfly" curves of heat flux versus pressure and shear for three acreage body points (10, 12 and 14) on the carbon fabric. Also shown on the butterfly plots are data point locations of arcjet testing reported below and at JSC [2]. As discussed, the arcjet data are plotted at heat fluxes 17.5 percent lower than those calibrated with cold-wall methods so they can be directly compared to the predicted hot-wall flight conditions.

\section{B. Arcjet Testing in Flight-Relevant Environments}

The BLAM test campaign in the Ames IHF was conducted at heat rates of $97 \mathrm{~W} / \mathrm{cm}^{2}$ (pressure of $5.63 \mathrm{kPa}$ ) and $136 \mathrm{~W} / \mathrm{cm}^{2}$ (pressure of $9.1 \mathrm{kPa}$ ). Previous tests at JSC [2] were conducted at $136 \mathrm{~W} / \mathrm{cm}^{2}$ (pressure of 3.35 $\mathrm{kPa}$ ) and $246 \mathrm{~W} / \mathrm{cm}^{2}$ (pressure of $9.6 \mathrm{kPa}$ ). These heat flux values are based on cold wall calibrations at locations where layer loss was observed. CFD analysis of the arcjet test conditions showed that a 17.5 percent reduction in measured cold-wall heat flux was necessary to account for hot-wall effects that are present in flight. Therefore, the cold-wall heat fluxes were reduced by this amount and plotted on the butterfly plots in Figure 2 to allow a direct comparison to the flight data. The CFD analysis shows that hot wall /cold wall effects have very little effect on pressure and shear in the arcjet tests. No adjustments for pressure were applied to the calibrated data. Although aerodynamic shear was not measured in the arcjet tests and CFD results for the BLAM and JSC tests were used to construct the plot shown at lower right of Figure 2. Comparison of the values for the arcjet test points to flight conditions clearly shows that arcjet testing to date is flight-relevant for use of the ADEPT entry system for the VITaL mission.

\section{BLAM Test Article, Instrumentation and Calibrations}

4

American Institute of Aeronautics and Astronautics 


\section{A. Test Article Design}

Wedge-shape test articles based on those used in previous design were used in the BLAM arcjet test campaign. A cross section of the wedge is shown in Figure 3. The wedges were water cooled, with integral carbon fabric supports made of graphite. Bi-axial loading of the fabric was applied via stainless steel clamps attached to stainless steel supports with tensioning bolts. Disc springs (Belleville washers) were used to maintain tension in the fabric to account for possible differential thermal expansion. The tensioning mechanisms are capable of applying flight-like loads on the fabric up to $131 \mathrm{kN} / \mathrm{m}$. The underside of the fabric was open to the innards of the wedge, a volume that was vented to the arcjet test chamber vacuum. The design was such that the outer surface of the fabric was flush with the outer mold line of the wedge, and the arcjet flow was incident to the wedge surface at an angle of 30 degrees. The top plate of the wedge was made from graphite and presented an overall flat plane to the flow. Grafoil was placed between the fabric and the graphite top plate to prevent archeated flow from entering the wedge. The top plates are not shown in Figure 3.

The requirement for bi-axial loading results in a very large wedge (for arcjet testing) with a span of $23.4 \mathrm{~cm}$. This large wedge size is a consequence of having built-in mechanisms for the loading, and it leads to limitations on heating rates that can be obtained in IHF simulations of entry heating. A wooden mock up of the wedge was successfully tested in the IHF to ensure that there was no blockage issue due to the model size. The area of fabric presented to the flow in this design was $7.62 \mathrm{~cm} \times 7.62 \mathrm{~cm}$.

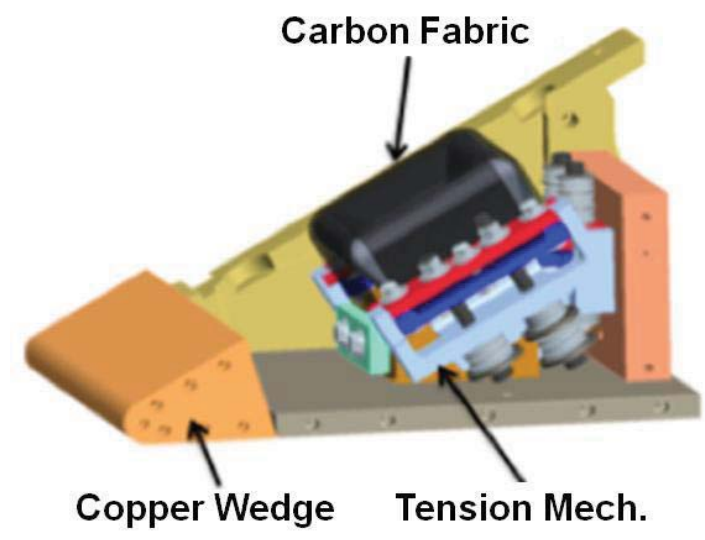

Figure 3. Cross sections of wedge used for the arcjet testing. Flow is from left to right and is incident to the cloth at an angle of 30 degrees. For all cases except two, the warp direction was oriented perpendicular to the flow direction.

\section{B. Instrumentation and Calibrations}

Key instrumentation for the BLAM tests included load washers and thermocouples. The load washers were located inside the wedges to ensure that their calibrations were not affected by heating effects. Thermocouples within the wedges were used to measure temperatures and ensure that the fabric tensioning parts were within pretest predictions, and not overheating. Other critical instrumentation included: (1) High definition video, that provided information on layer loss, (2) pyrometers to measure fabric surface temperatures at the locations where layer loss was observed, and (3) an IR camera to measure the global surface temperature distribution of the carbon fabric. Arcjet calibrations of the test conditions were made with a copper plate insert in place of the carbon fabric to measure heat flux and pressure at several locations on the surface of the wedge. These calibrations, which follow best practices for arcjet testing, were taken with the wedge in the flow for a short insertion time. For very short dwell times in the arc-heated stream, the copper calibration top plate does not heat appreciably and thus measurements are "cold wall" values.

\section{Test Results}

\section{A. Test Matrix}

The test matrix executed during the BLAM test campaign is shown in Table 1. Columns show the cold-wall heat fluxes, mechanical loading in the warp and weft directions on the fabric, insertion times and the observed times for removal of the top eight TPS layers of the fabric ( $\mathrm{LR}$ where $\mathrm{n}$ is the number of layers removed). For the BLAM tests, the acronym 8 LR is defined as the time it takes to remove the 8 TPS layers above the 4 structural layers. The edges of the eight fabric TPS layers near the clamps were severed to prevent any load 
redistribution through the un-ablated edges. Models B-1 and B-2 did not have cut edges because runs were required first to determine how much fabric was necessary to cut to force load in the fabric's center section. Model B-1 was the only uni-axial test conducted in the BLAM campaign. Model B-9 had the warp and weft directions switched

Table 1. Matrix for the BLAM Test Campaign and Times to Remove Eight Fabric Layers

\begin{tabular}{|c|c|c|c|c|c|c|}
\hline Run & Model & $\begin{array}{c}\text { C-W Heat } \\
\text { Flux } \\
\left(\mathrm{W} / \mathrm{cm}^{2}\right)\end{array}$ & $\begin{array}{c}\text { Warp Running } \\
\text { Load } \\
(\mathrm{N} / \mathrm{m})\end{array}$ & $\begin{array}{c}\text { Weft Running } \\
\text { Load } \\
(\mathrm{N} / \mathrm{m})\end{array}$ & $\begin{array}{c}\text { Insertion } \\
\text { Time } \\
(\mathrm{s})\end{array}$ & $\begin{array}{c}8 \text {-Layer } \\
\text { Removal } \\
(\mathrm{s})\end{array}$ \\
\hline 1 & B-1 & 97 & 65673 & 0 & 175 & 162 \\
\hline 2 & B-2 & 136 & 131345 & 65673 & 119 & 114 \\
\hline 3 & B-3 & 136 & 131345 & 65673 & 35 & N/A \\
\hline 4 & B-4 & 97 & 65673 & 32924 & 135 & N/A \\
\hline 5 & B-5 & 97 & 131345 & 65673 & 185 & 178 \\
\hline 6 & B-7 & 97 & 131345 & 65673 & 139 & N/A \\
\hline 7 & B-6 & 97 & 65673 & 32924 & 205 & 176 \\
\hline 8 & B-8 & 97 & 65673 & 32924 & 90 & N/A \\
\hline 9 & B-9 & 97 & 65673 & 131345 & 195 & 177 \\
\hline
\end{tabular}

\section{B. Mechanical Loads Experienced During Exposure to Aerothermal Heating}

As stated above, the first of the primary objectives of the BLAM testing described herein was to understand the ability of Weave SA to maintain structural integrity while under combined aerothermodynamic and mechanical loading. From all the BLAM testing, the most relevant data that address this objective are from models B-1, B5, and B-6 (see Table 1). Figure 4 (left) presents loading data as a function of time for these three runs. As can be seen from Figure 4 (left), there was minimal change to the load throughout the test time, and consequently the load carrying capability of the fabric. Thus, the structural integrity of the cloth while under flight-relevant combined heating and mechanical loads is most likely adequate for the current ADEPT design for the VITal mission. Furthermore, it is also shown that the removal of the top 8 TPS layers had little influence on the structural carrying capability of the fabric for loads up to the flight-relevant levels tested. Models B-5 and B-6 maintained load throughout the test, while loading in Model B-1 increased during testing. It is possible that uniaxial loading with uncut edges allowed load to be carried through the conditioned fabric. Graphite expands at a faster rate than that of the fabric and therefore the load cells carried more load, whereas in Models B-5 and B-6, with cut edges, the fabric strain release due to layer loss offset the graphite expansion during the test.
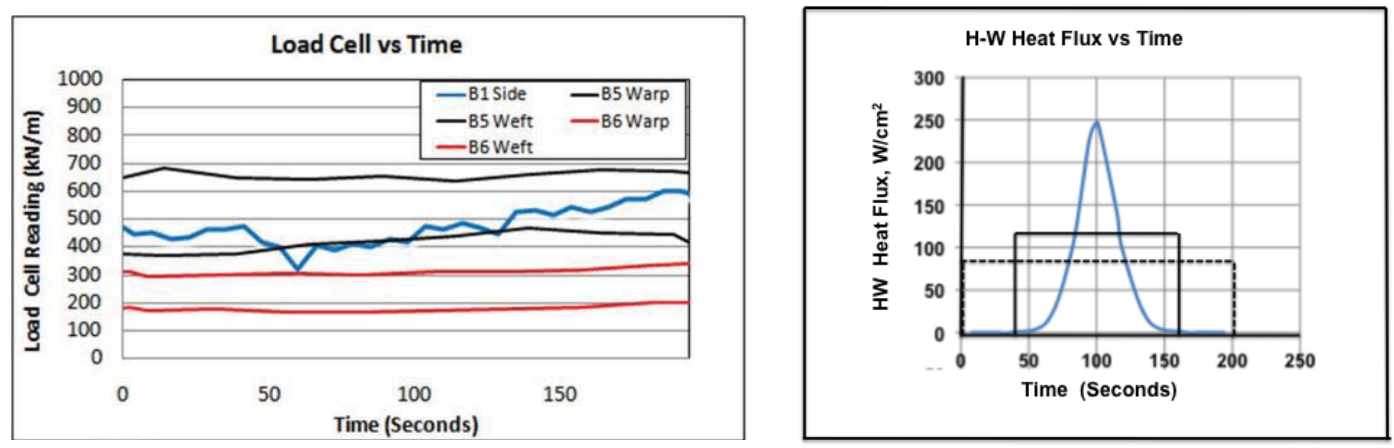

Figure 4: (Left) load cell readings from models B-1, B-5 and B-6 while subjected to arcjet heating. (Right). Hot -wall convective heating for VITaL trajectory and constant heating levels during BLAM B-2 and B-1, B-5 and B-6 runs.

6

American Institute of Aeronautics and Astronautics 
Importantly, the period that the cloth sustained the mechanical flight load in the arcjet testing was up to 205 seconds at $83 \mathrm{~W} / \mathrm{cm}^{2} \mathrm{H}-\mathrm{W}$ for models B-1, B-5 and B-6; and up to 119 seconds for model B-2 at a H-W heat flux of $116 \mathrm{~W} / \mathrm{cm}^{2}$. As can be seen from figure 4 (Right), the arcjet tests were at heating rates less than the peak during entry (blue curve), but the integrated heat loads in the tests exceed those VITaL will experience [1] in executing its deceleration from entry interface to payload separation at 230 seconds[1].

The secondary objective of the BLAM campaign to provide arcjet tested samples for microstructural analysis also been met. Tensile testing and scanning electron microscopy (SEM) of arcjet-tested fabrics from both the BLAM and JSC arcjet campaigns is underway and preliminary results have been reported [4]. The preliminary analysis suggests that the arcjet tested fabrics have tensile strengths nearly a factor of three greater than needed for flight. Preliminary SEM data show that fiber diameters in the top structural fabric layer exposed to the arcjet flow are thinned by oxidation, but no thinning is seen on the bottom-most structural layer. When completed, these results will be used in a thermal response margins policy to size the number of TPS sacrificial layers and for structural safety factors of the fabric in the final ADEPT-Vital flight design.

\section{Layer Loss During Combined Aerothermal and Mechanical Loads}

The second primary objective of the BLAM testing was to evaluate the rate of layer loss as a function of combined aerothermodynamics and mechanical loads. Data obtained from Models B-1, B-5, B-6 and B-9 address this objective. The measured rates of layer loss as a function of time are provided in Figure 5. The image on the left notionally depicts the eight TPS and four structural layers of the carbon fabric. The image on the right is a graph of layer loss versus time. When viewed together, which weave layer lost as a function of time during the test can be visualized. From the image, it is evident that the rate of layer loss is the same for all fabrics regardless of loading condition, i.e., the highly loaded biaxial fabric, the lower loaded biaxial fabric, and the highly loaded warp/weft flip direction fabric all result in the same rate of layer loss. Thus, biaxial load in the range of $33 \mathrm{kN} / \mathrm{m}$ to $131 \mathrm{kN} / \mathrm{m}$ had little or no impact on the rate of layer loss of the carbon fabric. These data also proved that flipping the warp and weft orientation with respect to the flow had little or no impact on rate of layer loss as a function of time. The final conclusion that can be drawn from this image is from Model B-1. The data from BLAM Model B-1 suggests that when the fabric is loaded uni-axially, with little load, the rate of layer loss is slightly increased for the remaining two TPS layers. It hypothesized that this increased rate of layer loss is due to an increased porosity of the fabric. However, future testing will provide more concrete evidence.

\section{Layer loss vs Time}
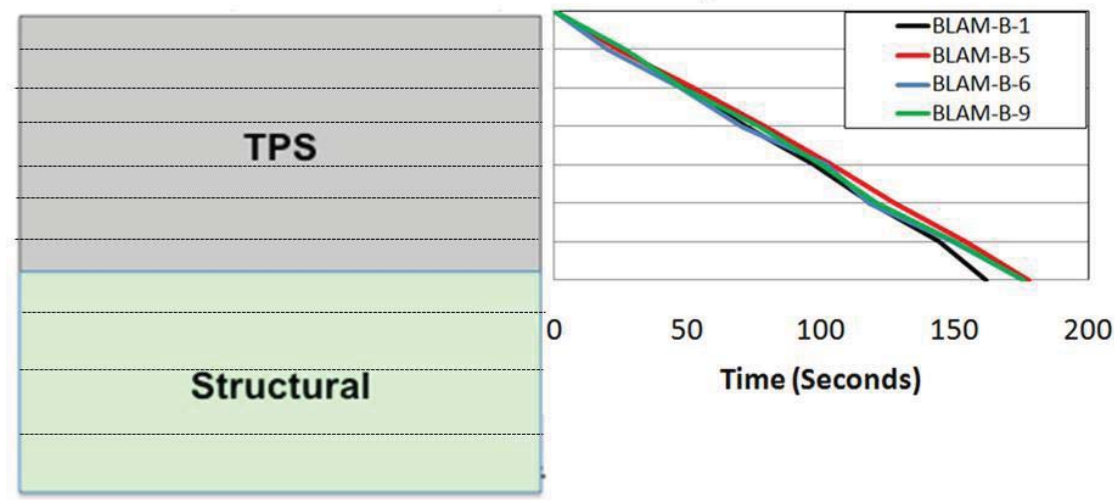

Figure 5: TPS layer loss measurements as a function of time. Model B-1 was in uni-axial tension.

\section{Comparison of Layer Loss Data to a FIAT-Based Thermal Response Model}

\section{A. FIAT-based Thermal Response Model for Weave SA Layer Removal}

Analysis [2] of the JSC test data and one test from the BLAM campaign provided the basis for a preliminary FIAT- based thermal response model that can be used to predict layer loss of woven carbon cloth. Further, that analysis suggests that for the tested conditions, diffusion-controlled oxidation dominates the thermophysics of layer removal. 
The Fully Implicit $\underline{\text { Ablation and }}$ Thermal (FIAT) response program is a one dimensional (1D), time-accurate thermal response solver for a TPS material undergoing thermal diffusion, surface ablation and internal pyrolysis [3]. FIAT is in widespread use to size TPS for ablative materials such as AVCOAT, PICA and SLA for NASA missions such as the Orion Crew Exploration Vehicle and the Mars Science Laboratory. FIAT fully accounts for the surface energy balance and can reliably predict surface and in-depth material temperatures as a function of time when anchored to arcjet data.

As reported in reference [2], Co-author B. Laub pioneered the use of FIAT to model layer removal for carbon fabrics. The approach for the fabric layer prediction is to run FIAT for a hypothetical solid carbon layer of the same thickness and density as the carbon fabric. Measurements of fabric layer removal rates are roughly twice the FIAT predictions of recession rates for the equivalent carbon slab [2]. Equation (1) from [2] is used to match the FIAT predictions for each test result by adjusting the value of the recession augmentation $(R A)$ term. It has been surmised [2] that the recession augmentation factor accounts for the greater surface area of the fabric as compared to that of the hypothetical solid carbon layer. The FIAT- based model predicts the carbon fabric layer removal rate $S_{\mathrm{dot}}$ to be given by:

$$
S_{\mathrm{dot}}=(1+R A) \times B^{{ }^{\prime}} \times C_{H} / \rho
$$

Here $R A$ is a recession augmentation parameter, $B^{\prime}{ }_{C}$ is a non-dimensional out-going mass flux of carbon divided by the incoming free stream mass flux, $C_{H}$ is the convective heating film coefficient (equal to the convective heat rate $\mathrm{q}_{\mathrm{c}}$ divided by the difference of the boundary layer edge and the wall enthalpies) and $\rho$ is the fabric density. For diffusion-controlled oxidation of carbon in air, $B^{\prime}{ }_{c}$ is fixed at a constant value of 0.176 , and as can be seen in Figure 5 is independent of surface pressure over a wide range of temperatures $\left(\sim 1000\right.$ to $\left.2800{ }^{\circ} \mathrm{K}\right)$. Thus, for this model with a fixed value of cloth density, removal of the carbon layers solely depends on convective film coefficient. As reported in Arnold et al [2], it appears that a recession augmentation of $R A=0.9 \pm 0.2$ nearly correlates all of the JSC data at two different heating rates and the results from one test obtained during the BLAM campaign. Note that a value of $R A=0.9$ means that the carbon fabric layer removal rate is 1.9 times faster than the hypothetical carbon layer of the same thickness and density. Because both the BLAM and JSC arcjet tests were conducted in air, $B^{\prime}{ }_{c}(0.176)$ or air is the appropriate quantity to use in equation (1).

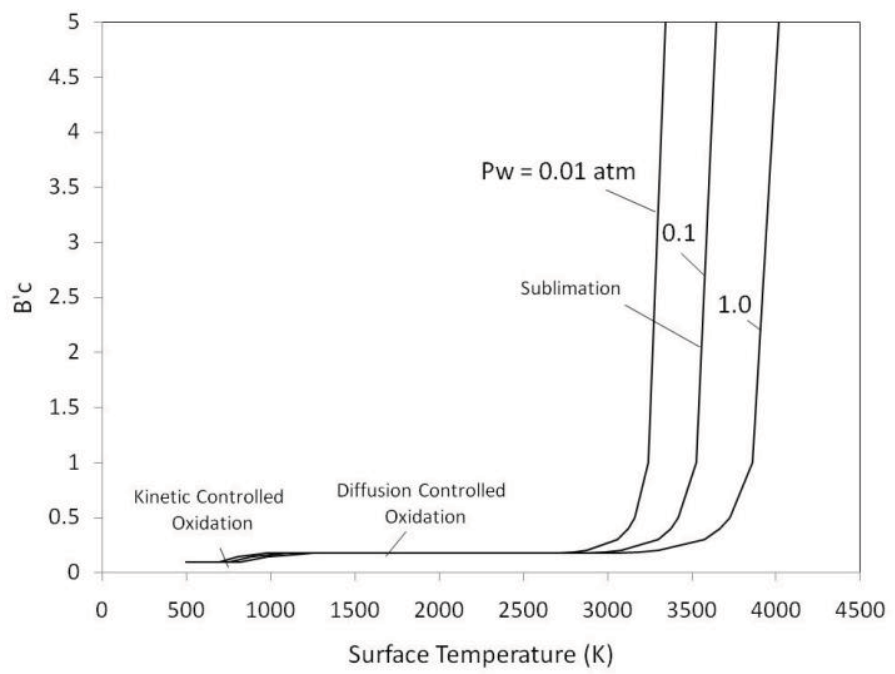

Figure 6. $B{ }_{C}$ for air. This parameter is invariant of pressure over a significant range of temperatures from $\sim 1000$ to $2900{ }^{\circ} \mathrm{K}$ in the diffusion-controlled oxidation regime.

\section{B. Comparison of the FIAT-based Thermal Response Model to Test Data and Model Refinement}

Focus is now placed on comparisons of the measured data for surface temperatures and layer removal to predictions from the preliminary thermal response model [2]. The free stream predictions required for the wedge aerothermodynamic performance, including the convective heat transfer coefficient discussed above, were determined from CFD calculations of the arcjet nozzle flow field and sampling at the test location [5]. The assumption of a uniform flow field at the test location is an approximation, but was deemed sufficient for the present study. 
The first case is for model B-2 taken at a cold wall heat flux of $136 \mathrm{~W} / \mathrm{cm}^{2}$ and a pressure of $9.1 \mathrm{kPa}$.

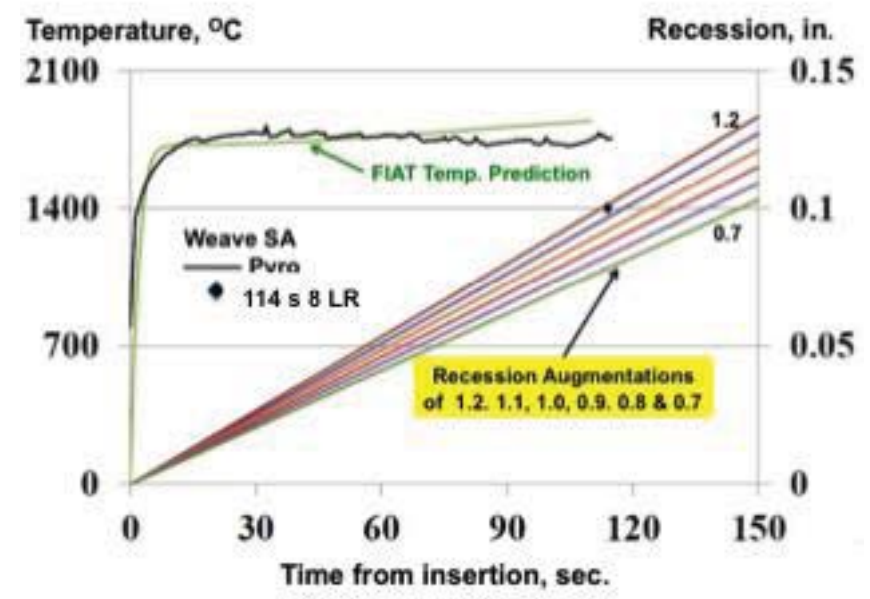

Figure 7. Comparison of measured temperatures and fabric layer removal rates on model B-2 to those from the FIATbased thermal response model. The BLAM test conditions were $136 \mathrm{~W} / \mathrm{cm}^{2}$ cold wall at $9.1 \mathrm{kPa}$. Temperature to be read on the left scale and recession in inches on the right. The Weave SA 12-layer fabric thickness is $0.38 \mathrm{~cm}(0.15$ "). A $R A$ value for this test to match the measurement to the FIAT-based prediction for the removal of 8 TPS layers is slightly less than 1.2

As can be seen in Figure 7, the agreement between the predicted temperatures and those measured by pyrometery is excellent. The "fan" of FIAT predicted recession corresponds to a set of $R A$ values starting at 1.2 and going down in increments of 0.1 to 0.7 . Comparison of the recession data for eight layer removal $(8 \mathrm{LR})$ to the recession predictions at $0.254 \mathrm{~cm}(0.1 \mathrm{inch})$ shows that the model B-2 $8 \mathrm{LR}$ data is matched with a $R A$ value of slightly less than 1.2 .

Figure 8, taken from Arnold et al [2] is from the JSC arcjet testing at a C-W heat flux of $136 \mathrm{~W} / \mathrm{cm}^{2}$ and a pressure of $3.35 \mathrm{kPa}$. The heat flux is identical to that of model B-2, at Ames, but the pressure is lower by nearly a factor of three.

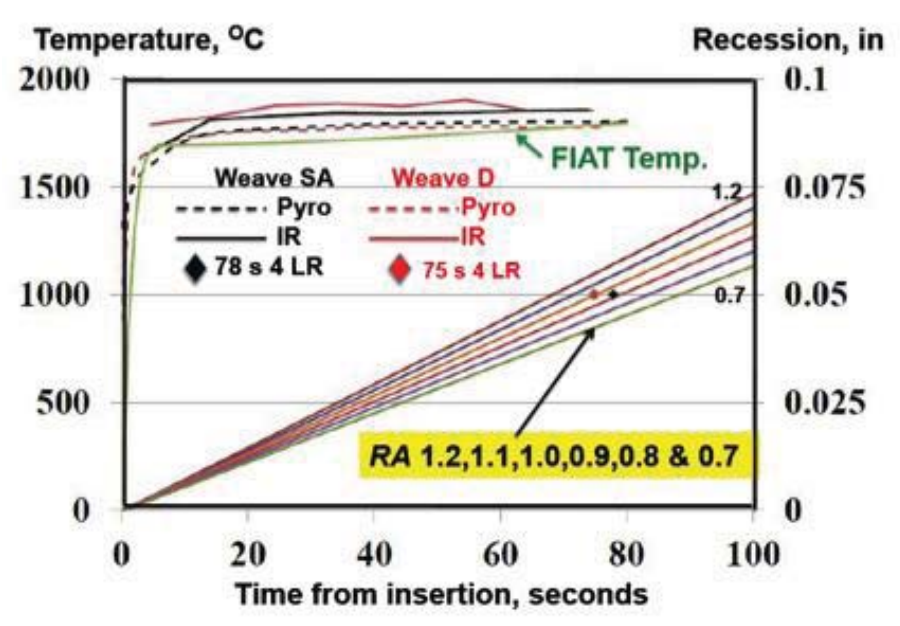

Figure 8. Carbon layer removal taken from [2]. Conditions for this JSC arcjet test were $136 \mathrm{~W} / \mathrm{cm}^{2}$ (cold wall) and a pressure of $3.35 \mathrm{kPa}$. These fabrics had a total of 8 layers and were 0.1 " thick. The Weave D fabric is very similar to the eight-layer Weave SA so it should exhibit layer removal rates like those observed for Weave SA. The RA value required to match the observed 4 TPS layer removal were slightly more than 0.9 for for Weave SA and 1.0 for Weave D. 
The pyrometer data shown by the dashed lines for two tests are in close agreement with the FIAT prediction based on an assumed cloth emissivity of 0.9. As noted in Arnold et al [2], temperatures measured in the JSC facility with the IR camera were normally about $100{ }^{\circ} \mathrm{C}$ higher than pyrometric measurements because of an issue with scattered light in the IR camera and so this difference is understood. Inspection of recession data shows that $R A$ values of 0.9 to 1.0 match the FIAT predictions for the removal of the four top TPS layers (4 LR) of the eight-layer fabric of Weaves SA and D. The FIAT-based correlation of the JSC and BLAM data with similar values of $R A$ at a $\mathrm{C}-\mathrm{W}$ heat rate of $136 \mathrm{~W} / \mathrm{cm}^{2}$ to FIAT for widely different pressures (3.35 to $9.1 \mathrm{kPa}$ ) suggests that the dominant phenomenon in the testing is diffusion-controlled oxidation.

Figure 9 depicts test data from Models B-1, B-5 and B-9. The test condition here was $97 \mathrm{~W} / \mathrm{cm}^{2} \mathrm{C}-\mathrm{W}$ at a pressure of $5.63 \mathrm{kPa}$. Again, temperatures obtained by pryometry and temperature predicted by FIAT compare very well. Comparing measured recession through the eight layers of TPS, a RA value of 0.9 matches the FIAT prediction to the measured layer removal rates for Models B-5 and B-6. However, the results for the uni-axial loaded test with Model B-1 show a higher rate of layer removal. While not completely understood, it is noted that the recession for this single test is still "in family" with data from both the JSC and BLAM test results.

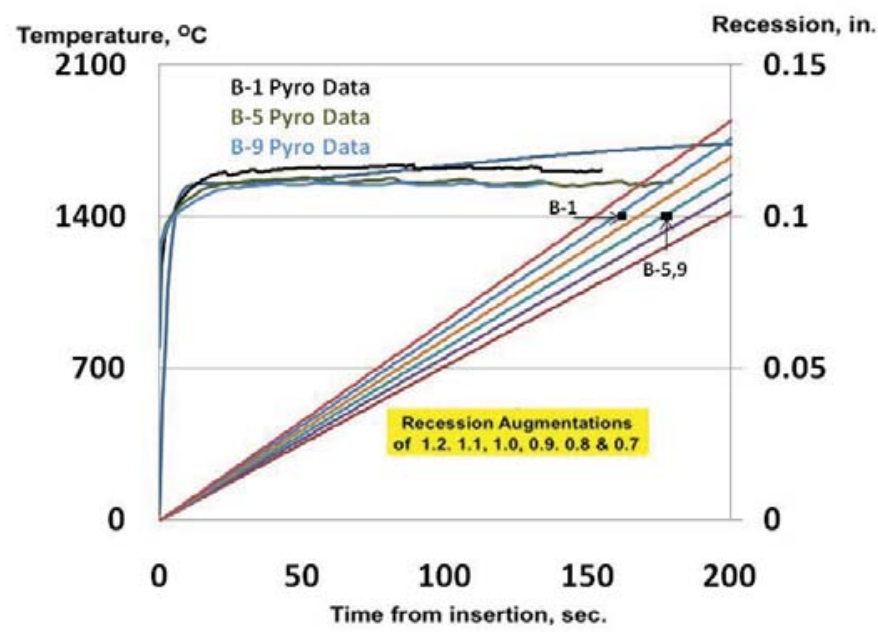

Figure 9. Comparison of FIAT predictions to data from BLAM Models B-1, B-5 and B-9. Conditions for these tests are $97 \mathrm{~W} / \mathrm{cm}^{2}$ cold wall at a pressure of $5.63 \mathrm{kPa}$. Model B-1 was unique in the BLAM test series because it was under uni-axial loading. Fabric thickness here is $0.38 \mathrm{~cm}(0.15$ ").

From Arnold et al [2], a single data point in the JSC testing was obtained at $246 \mathrm{~W} / \mathrm{cm}^{2} \mathrm{C}-\mathrm{W}$ and a pressure of $9.6 \mathrm{kPa}$. It was shown that a $R A$ value of 0.7 was required to match the FIAT prediction for recession to the observed layer removal.

It is remarkable that the arcjet data for carbon cloth layer removal from different facilities, test articles, and fabric thicknesses obtained at widely different heat fluxes and pressures can be correlated with the FIAT model described above. This observation, and the fact that the measured rates for carbon cloth layer removal are roughly a factor of two higher than FIAT predicts for a hypothetical layer of solid carbon reinforces the notion that the root cause of the difference is because of the surface area of cloth as compared to that of a solid layer.

Runs with the FIAT program were performed [2] to evaluate the relationship of the uncertainty of the arcjet heat flux to the deduced recession augmentation. This analysis suggests that at a heat flux of $246 \mathrm{~W} / \mathrm{cm}^{2}$ and assuming that the true recession augmentation is 0.9 , an uncertainty of \pm 10 percent in the heat flux results in a variation of $R A$ from 1.1 to 0.75 . It is estimated that the measured time for burn-through for the top TPS layers has an uncertainty of about plus-or-minus one second. Realizing that arcjet data normally exhibit scatter of at least \pm 10 percent, it is probable that a relatively large set of arcjet data will be required for a statistically significant determination of the overall recession augmentation for the carbon cloth layer removal. Pressure is not a significant factor in uncertainty, provided diffusion-controlled oxidation is the dominant process in the testing.

Thus, correlations to date from both the BLAM data and that from the JSC testing suggest that the preliminary FIAT-based model using an $R A$ value of 0.9 can be used for predictions of layer removal and surface temperatures at conditions tested in air between 97 and $246 \mathrm{~W} / \mathrm{cm}^{2}$. As stated above, this is significant because the data were obtained for ADEPT entry system components at flight-relevant conditions for the VITaL mission.

10

American Institute of Aeronautics and Astronautics 
Further, it appears that FIAT code can be used as a preliminary, predictive thermal response model for missions flight designs if the $B_{C}^{\prime}$ curves were modified to account for Venus' $\mathrm{CO}_{2}-\mathrm{N}_{2}$ atmosphere [6]. Finally, it is suggested that predictions of the bounds for layer removal in air can be made by using limits on RA of $0.9 \pm 0.2$.

\section{Summary}

The BLAM test campaign was quite successful in meeting its objectives as follows:

The primary test objectives of the BLAM arcjet test campaign were:

1. To evaluate the Super Weave A capability to maintain structural integrity under combined flight-like aerothermodynamic and flight-like bi-axial tensile loading. The BLAM arcjet testing at NASA Ames has shown that the ADEPT carbon fabric is capable of performing its TPS and structural functions under flight-like aerothermodynamic and bi-axial structural loads relevant to the entry conditions anticipated for the VITaL mission.

2. To evaluate the rate of the fabric layer loss as a function of different aerothermal and biaxial loadings. The BLAM arcjet testing campaign has met its objective to provide information on the rate of layer removal (recession) of the carbon fabric while in flight-like combined aerothermodynamic and mechanical (tensile) loading. The data shows that there is essentially no effect of bi-axial loading (or the warp-weft fabric orientation) on layer removal over relevant ranges for the ADEPT flight design for the VITaL mission.

Secondary test objectives were:

To evaluate the residual load bearing capacity of the arcjet-tested material and to examine the microstructure of the arcjet-tested material using Scanning Electron Microscopy (SEM):

Samples from both the BLAM and JSC arcjet test campaigns are currently being analyzed and Preliminary results have been published [4].

This paper goes beyond the stated primary objectives of the BLAM test campaign because the data from testing on layer removal have been used to extend the range of the preliminary FIAT-based thermal response model [2] to predict layer removal as discussed in Section VI. It appears that a recession augmentation $R A$ of 0.9 could be used for thermal response to predict layer removal in future arcjet testing in air from 97 to $246 \mathrm{~W} / \mathrm{cm}^{2}$ across a wide pressure range. Based on performance arcjet testing in flight-relevant combined aerothermodynamics shear environments, it seems safe to conclude that Weave SA is viable from the perspective of its use for acreage TPS. Because of the limited number of tests conducted to date, the proposed FIAT-based thermal response model must be considered to be preliminary. It is possible to modify the values of $B_{c}{ }_{c}$ by analytical methods [6] and use the model to predict layer removal during a typical trajectory in a $\mathrm{CO}_{2}$ rich atmosphere.

\section{Forward Work}

While the work discussed in this paper provides a sound basis for claiming that the 3D Weave SA carbon fabric is viable for use on the ADEPT entry system for the VITaL mission, considerable arcjet testing and analysis remains to be accomplished before the cloth could be used in a final mission design. In the near term, all of the BLAM test data can be used to add to the layer removal database at 97 and $136 \mathrm{~W} / \mathrm{cm}^{2}$. This can be accomplished by conducting computed tomography on those articles that did not have all the TPS layers removed to the structural layer to determine how many layers were actually removed during the time the test article was kept in the arcjet stream. Preliminary work conducted at NASA Ames has shown this is a viable approach that will add to our understanding of the scatter in the arcjet data.

Only a single data point at the highest heating rate of $246 \mathrm{~W} / \mathrm{cm}^{2} \mathrm{C}-\mathrm{W}$ as been obtained at JSC. Owing to the size of the copper wedge required for the bi-axial loading, the maximum heat rate that can be obtained with that test article in Ames IHF is $136 \mathrm{~W} / \mathrm{cm}^{2}$. More testing at the $246 \mathrm{~W} / \mathrm{cm}^{2}$ conditions should be obtained on wedges with combined heating and shear at JSC or in the new Test Point 3 (TP3) Facility at NASA Ames. Based on the data in hand, it appears that aerodynamic shear is not a failure mode for the carbon fabric, at least for the acreage areas. Testing using a stagnation model approach should he undertaken to add more data at even higher heating rates in addition to testing at several levels of constant heat flux across a broad range of pressure to validate the current evidence that diffusion-controlled oxidation dominates the thermophysics. Clearly, data need to be obtained at lower heat fluxes and pressure scans at those conditions to complete the database needed to improve the fidelity of the preliminary FIAT-based thermal response model for Weave SA. Testing should also be

11

American Institute of Aeronautics and Astronautics 
conducted with $\mathrm{CO}_{2}$-rich test gases so that the model can be validated for Venus missions, and as a bonus for those for potential ADEPT missions to Mars.

The paragraphs above address the desired work for completeness of test and analysis for Weave SA for use on acreage. Similar work needs to be done in the areas where the fabric is interfaced with the TPS on the ADEPT rigid nose and at the fabric-rib interfaces where problems akin to gap heating in rigid TPS may be important. Finally, testing needs be done to better understand the effects of combined heating (albeit lower than for acreage) and higher shear that will be present on the trailing edge of the ADEPT entry system.

\section{IX.Acknowledgments}

Keith Peterson was supported by Contract NNA10DE12C from the Entry Systems and Technology Division to ERC, Inc. The authors wish to acknowledge the BLAM team - Dinesh Prabhu, Paul Wercinski, Sev Rosario, Tom Squire, Paul Banicevic, Peter Gage, Carl Kruger and Ethiraj Venkatapathy. Without their untiring support, this testing would not have been successful. The authors owe a debt to the late Bernard Laub, who pioneered the development of the concept of using a FIAT-based thermal response model for solid carbon to understand and predict the layer removal of 3-D woven carbon fabrics. We appreciate the contributions of Parul Agrawal in evaluating the microstructure of the arcjet tested fabric samples and look forward to future collaborations with her. We thank Y-K Chen's for all the FIAT predictions. The BLAM test campaign could not have been accomplished without the outstanding efforts of Ames arcjet test crew led by Imelda Terrazas-Salinas and Enrique Carballo. Finally, we greatly appreciate the efforts Dinesh Prabhu for his critical review of this paper and Nancy Mangini for preparing this manuscript for publication.

\section{References}

[1] Smith, B., Venkatapathy, E., Wercinski, P., Yount, B., Prabhu, D., Gage, P., Glaze, L., and Baker, C., "Venus In-Situ Explorer Mission Design using a Mechanically Deployed Aerodynamic Decelerator", Big Sky IEEE Aerospace Conference, Big Sky Montana, March 2013.

[2] Arnold, J. O., Laub, B., Chen, Y.-K., Prabhu, D. K., Bittner, M. E., and Venkatpathy, E., "Arcjet Testing of Woven Carbon Cloth for Use on Adaptive Deployable Entry Placement Technology", International Planetary Probe Workshop Number 10, San Jose State University, San Jose California June 17-21, 2013.

[3] Chen, Y.-K. and Milos, F. S., "Ablation and Thermal Response Program for Spacecraft Heatshield Analysis," Journal of Spacecraft and Rockets, Vol. 36, No. 3, pp 475-483, 1999.

[4] Agrawal, P., Pham, J. T. Arnold, J. O., Peterson, K. H., and Venkatapathy, E., "Micromechanical Characterization and Testing of Carbon Based Woven Thermal Protection Materials, Cocoa Beach Conference, Cocoa Beach, FL, January 2013.

[5] Prabhu, D. K., Saunders, D., Tang, C., Terrazas-Salinas, I., Carballo, J. E., and Driver, D., "CFD Analysis Framework for Arc-Heated Flowfield, II: Shear Testing in Arc-jets at NASA ARC," AIAA Paper 2009-4081, AIAA Thermophysics Conference, Chicago, IL, June 2009.

[6] Milos, F. S., Private communication, February 2013. 